\title{
Identidad e integración social: análisis de la sociabilidad en una sociedad de socorros mutuos (1902-1933)
}

\section{Pilar León Sanz (*)}

(*) orcid.org/0000-0003-0247-6101. Historia de la Ciencia, Universidad de Navarra. mpleon@unav.es

Dynamis

[0211-9536] 2015; 35 (2): 409-431

http://dx.doi.org/10.4321/S0211-95362015000200007
Fecha de recepción: 17 de marzo de 2014

Fecha de aceptación: 4 de febrero de 2015

SUMARIO: 1.- Introducción. 2.-La Sociedad de Socorros Mutuos La Conciliación. 3.-Las fuentes. 3.1.-Entrega de ropitas a las madres más pobres. 3.2.- La fiesta de La Conciliación. 4.- Un análisis desde la historia de las emociones. 4.1.-Comunicar y movilizar. 4.1.1.—Las conferencias de las fiestas. 4.2.-Escenificar. 4.2.1.—Los lugares de celebración. 4.3.—Reglamentar. 5.-El carácter transformador de las emociones.

RESUMEN: El artículo estudia la sociabilidad en las fiestas y en las celebraciones del Consultorio de Niños de Pecho organizadas periódicamente por la sociedad de socorros mutuos La Conciliación entre 1902 y 1933. Eran actos recreativos que también tenían una finalidad educativa. La Conciliación (1902-1984) fue fundada en Pamplona, con carácter mixto y católico, exclusivamente masculina hasta 1936. Su principal actividad fue la mediación laboral y la asistencia en la enfermedad a los socios obreros y sus familias, a través de un subsidio económico y del servicio médico. A partir de las noticias publicadas en la prensa y de los documentos del archivo de la sociedad de socorros mutuos, se describen los acontecimientos y se analizan las prácticas emocionales de las celebraciones. Los actos del Consultorio de Niños de Pecho suscitaron emociones positivas que estimularon los sentimientos maternales, de piedad y caridad, o de utilidad social de las clases acomodadas. En las fiestas de La Conciliación se reconocen los aspectos emocionales relacionados con la comunicación, la movilización y la regulación: las conferencias y los actos proponían a los socios obreros y a sus familias un modelo social y una concepción ideológica y religiosa. El carácter performativo de las emociones reforzó la identidad de los miembros y su integración social en la ciudad. Se trató de una sociabilidad al servicio del proyecto de La Conciliación.

PALABRAS CLAVE: Sociabilidad, sociedades de socorros mutuos, España, siglo XX, historia de las emociones.

KEY WORDS: Sociability, mutual aid societies, Spain, twentieth century, history of emotions. 
In memoriam Juan Antonio Paniagua $(\dagger)$

\section{Introducción $(*)$}

Son numerosas las líneas de trabajo abiertas sobre el estudio de las emociones que tratan de recuperar la llamada historia de la subjetividad. A través del análisis de las motivaciones e intenciones que explican las conductas o los acontecimientos, proponen escribir la historia de «dentro a fuera» (the inside out) ${ }^{1}$. Desde esta perspectiva, pretendemos revisar la sociabilidad originada a través de las fiestas (actividades educativas y de ocio), organizadas periódicamente por la Sociedad Protectora de Obreros La Conciliación desde su fundación en 1902 hasta $1933^{2}$.

Peter N. Stearns y Carol Z. Stearns han demostrado la validez de este acercamiento para la comprensión de la historia de EE.UU. a lo largo de los siglos XIX y XX $\mathrm{XX}^{3}$. Por su parte, Christian Bailey explica que las emociones

$\left({ }^{*}\right)$ La investigación se inició con el proyecto «La sociedad de obreros La Conciliación: escenario de prácticas médicas (1902-1984)», PIUNA (2006-09); forma parte del proyecto «Cultura emocional e identidad», Instituto de Cultura y Sociedad, Universidad de Navarra. Un resumen de la investigación fue presentado en el VII Congreso de Historia Social, Madrid, 24-26 de octubre de 2013.

1. Matt, Susan J. Current emotion research in History: Or, doing history from the inside out. Emotion Review. 2011; 3 (1): 117-124 (118). Una revisión historiográfica de la historia de las emociones: Plamper, Jan. The history of emotions: An interview with William Reddy, Barbara Rosenwein, and Peter Stearns. History and Theory. 2010; 49: 237-265; Pampler, Jan. Historia de las emociones: caminos y retos. Cuadernos de Historia Contemporánea. 2014; 36: 17-29. Ute Frevert destaca los aspectos culturales y sociales de lo emocional: Frevert, Ute. Defining emotions: Concepts and debates over three centuries. In: Emotional lexicons. Continuity and change in the vocabulary of feeling 1700-2000. Oxford: Oxford University Press; 2014, p. 1-31 (25).

2. Maurice Agulhon inició el estudio histórico de la sociabilidad (La Sociabilité méridionale. Confréries et associations en Provence orientale à la fin du XVIIIe siècle. Aix-en-Provence: La Pensée Universitaire; 1966). Guereña, Jean-Louis. Espacios y formas de la sociabilidad en la España contemporánea. Introducción. Hispania. 2003; 214: 409-414, analiza la re-definición del concepto de Agulhon que integra tanto aspectos formalizados (como la vida asociativa, caso de este artículo) como otros menos estructurados de la vida cotidiana (p. 412). También Navarro Navarro, Javier. Mundo obrero, cultura y asociacionismo: algunas reflexiones sobre modelos y pervivencias formales. Hispania. 2003; 214: 467-484.

3. Stearns, Peter N. An emotional history of the United States. New York: New York University Press; 1998; Stearns, Peter N. American cool: constructing a twentieth-century emotional style. New York: New York University Press; 1994; Stearns, Carol Z. Anger: the struggle for emotional control in America's history. Chicago: University of Chicago Press; 1986. 
interpersonales son normalmente analizadas como agentes de sociabilidad ${ }^{4}$. Sin embargo, otros historiadores, como William Reddy, han señalado que abordar de esta manera los cambios históricos tiene una dificultad mayor de la que se había considerado inicialmente ${ }^{5}$. Uno de los mayores problemas de la historia de las emociones es encontrar fuentes adecuadas para saber lo que la gente «sintió» en un tiempo pasado, en el que las emociones se entendían y expresaban de manera diferente a la actual ${ }^{6}$.

Describimos en primer lugar las características de La Conciliación y el contexto local en el que se desarrolló, como paso previo al análisis de la sociabilidad en las fiestas organizadas por La Conciliación desde el punto de vista de la historia de las emociones. Se trata de una investigación que quiere sumarse a los estudios sobre la sociabilidad en las sociedades de socorros mutuos (SSM) y mutualidades ${ }^{7}$.

4. Bailey distingue entre las emociones que sienten las personas de una comunidad (nación, clase, asociación), de las emociones de los individuos en una masa. Bailey, Christian. Social emotions. In: Frevert, n. 1, p. 201-229 (202).

5. Reddy, William M. Historical research on the self and emotions. Emotion Review. 2009; 1 (4): 302-315 (304-306). Dificultad comentada por el propio Stearns en Matt, Susan J.; Stearns, Peter N, ed. Doing emotions history. Urbana: University of Illinois Press, 2014.

6. Matt, n. 1, p. 119; Reddy, n. 5, p. 312.

7. Guereña, Jean-Louis. Sociabilidad, cultura y educación en Asturias bajo la Restauración (18751900), Oviedo: Real Instituto de Estudios Asturianos; 2005, p. 77-88, 115-142; Guereña, JeanLouis. Hacia una historia socio-cultural de las clases populares en España (1840-1920). Historia Social. 1991; 11: 149-151; Motilla Salas, Xavier. Bases bibliográficas para una historia de la sociabilidad, el asociacionismo y la educación en la España contemporánea. Historia de la Educación: Revista Interuniversitaria. 2012; 31: 339-358; Ralle, Michel. La sociabilidad obrera en la sociedad de la Restauración (1875-1910). Estudios de Historia Social. 1989; 50-51: 161199; Maza Zorrilla, Elena, coord. Asociacionismo en la España contemporánea: vertientes y análisis interdisciplinar. Valladolid: Universidad de Valladolid; 2003; Canal, Jordi. Historiografía y sociabilidad en la España contemporánea: Reflexiones con término. Vasconia. 2003; 33: 11 27. Estudian la sociabilidad en ámbitos locales: Arias Fernández, Modesto. El asociacionismo en Puertollano: el caso de la Sociedad Benéfica La Esperanza. In: Sánchez Sánchez, Isidro; Villena Espinosa, Rafael; Guereña, Jean-Louis. Sociabilidad fin de siglo: espacios asociativos en torno a 1898. Cuenca: Universidad de Castilla-La Mancha; 1999, p. 225-240; Brey, Gérard. Aproximación a la sociabilidad popular en las ciudades gallegas (1833-1914). Estudios de Historia Social. 1989; 50-51: 223-242; Guereña, Jean-Louis. Una aproximación a la sociabilidad popular: el caso de Asturias bajo la Restauración (1875-1900). Estudios de Historia Social. 1989; 50-51: 201-222; Morales, Manuel. La sociabilidad popular en Málaga, 1840-1874: de la tutela burguesa a la afirmación de una identidad diferenciada. Estudios de Historia Social. 1989; 50-51: 243-271. 


\section{La Sociedad de Socorros Mutuos La Conciliación}

En España, hasta la década de 1940, las SSM y las mutuas tuvieron un protagonismo relevante en la provisión de la asistencia médica y otros beneficios sociales para un alto porcentaje de trabajadores y sus familias ${ }^{8}$. La Sociedad Protectora de Obreros La Conciliación, fundada en Pamplona en 1902, fue, hasta 1936, la SSM más importante de Navarra ${ }^{9}$, puesto que el número de obreros asociados se mantuvo por encima de los 1.000. Hasta esa fecha, La Conciliación incluyó asociaciones de trabajadores por cada oficio: albañiles, carpinteros, cerrajeros, tejedores, canteros, zapateros, peones, labradores, servicios diversos, sastres, dependientes, panaderos, tipógrafos, pintores y chocolateros, etc ${ }^{10}$.

Inicialmente, La Conciliación fue una sociedad de socorros mutuos mixta, formada por obreros, patronos y protectores que siguió las características promovidas por el catolicismo social de comienzos del siglo XX. A lo largo de los años se convirtió en una asociación de sociedades de trabajadores. A partir de 1933, dejó de tener carácter confesional y se constituyó en mutualidad, unida a una veintena de sociedades obreras de diversos oficios. En diciembre de 1936, La Conciliación se fusionó con la pamplonesa Mutualidad de Obreros Profesionales, lo que supuso más cambios en el número y tipo de socios. Tras la Guerra Civil, La Conciliación, como otras instituciones semejantes, perdió uno de sus objetivos principales: la participación en las cuestiones laborales; sin embargo, aun con una tendencia decreciente, continuó prestando asistencia médica y diversos subsidios hasta 1984, fecha en que desapareción ${ }^{11}$.

8. León Sanz, Pilar. Professional responsibility and the welfare system in Spain at the turn of the 19th century. Hygiea Internationalis. 2006; 1 (5): 75-90; Guereña, 2005, n. 7, p. 5-88; VilarRodríguez, Margarita; Pons-Pons, Jeronia. The introduction of sickness insurance in Spain in the first decades of the Franco dictatorship (1939-1962). Social History of Medicine. 2013; 26 (2): 267-287.

9. Coinciden con esta apreciación: Andrés-Gallego, José. Pensamiento y acción social de la Iglesia en España. Madrid: Espasa-Calpe; 1984, p. 287; Goñi Gaztambide, José. Historia de los obispos de Pamplona. Pamplona: Eunsa; 1999, vol. 11, p. 561-599; Pejenaute Goñi, Javier M. Las Sociedades de Socorros Mutuos en Navarra (finales del siglo XIX-comienzos del XX). Congreso de Historia de Euskal Herria. Vitoria: Editorial Txertoa Argitaldaria; 1988, vol. 6, p. 279-281.

10. Un estudio global de la Sociedad en: León-Sanz, Pilar. Medical assistance provided by La Conciliación, a Pamplona Mutual Assistance Association (1902-1984). In: Harris, Bernard, ed. Welfare and old age in Europe and North America: The development of social insurance. London: Pickering and Chatto; 2012, p. 137-166.

11. León-Sanz, n. 10. 
Durante el periodo aquí estudiado, La Conciliación fue una institución masculina en la que los socios obreros y sus familiares directos eran los únicos receptores de los servicios ofertados. En investigaciones previas hemos establecido las condiciones y la evolución de los beneficios ofrecidos por La Conciliación, que fueron semejantes a los ofertados por otras SSM y mutuas coetáneas: la mediación laboral, la asistencia en la enfermedad a los socios obreros y sus familias, a través de un subsidio económico y del servicio médico (desde 1902) y farmacéutico (desde 1910), la caja para crónicos (desde 1914) y el servicio de comadrona (desde 1914); una ayuda económica post-mortem (desde 1918); cooperativa (entre 1912 y 1922) ${ }^{12}$. También hemos estudiado las relaciones que la Sociedad estableció con los profesionales de la salud ${ }^{13}$; y ha resultado especialmente interesante analizar la percepción de los socios y sus familias acerca del servicio médicofarmacéutico recibido en la Sociedad ${ }^{14}$.

Por la importancia que tiene para el estudio de las dinámicas asociativas, interesa destacar que La Conciliación se desarrolló en un ámbito exclusivamente urbano. Durante los años aquí considerados, Pamplona era una pequeña ciudad con una población que se estabilizó en torno a los 30.000 habitantes ${ }^{15}$. Como en otras provincias españolas, también aquí existió un grave problema social derivado de la falta de empleo. La segunda década

12. León-Sanz, n. 10.

13. León Sanz, Pilar. La concertación de la asistencia en la enfermedad en La Sociedad de Obreros La Conciliación (1902-1919). In: Navarra: Memoria e Imagen. Pamplona: Eunate; 2006, vol. 2, p. 97-108; León Sanz, Pilar. The Mutual Benefit Societies' responses to the 1918-19 influenza pandemic in Pamplona. In: Porras, María Isabel; Davis, Ryan A. Emerging infection, emergent meanings: The Spanish influenza pandemic of 1918-1919. Rochester: University of Rochester Press; 2014, p. 152-170.

14. Leon Sanz, Pilar. From claims to rights: Patient complaints and the evolution of a Mutual Aid Society (Sociedad Protectora de Obreros La Conciliación, Pamplona, 1902-1936). In: Reinarz, Jonathan; Wynter, Rebecca, eds. Complaints, controversies and grievances in medicine: Historical and social science perspectives. London: Routledge; 2015, p. 109-128; León Sanz, Pilar. Los relatos de los enfermos: análisis de las quejas de los socios en una Sociedad de Socorros Mutuos (1902-1919). In: Ortiz Gómez, Teresa; Olagüe de Ros, Guillermo; Rodríguez Ocaña, Esteban, coords. La experiencia de enfermar en perspectiva histórica. Granada: Editorial Universidad de Granada; 2008, p. 51-54.

15. Floristán Samanes, Alfredo, dir. Gran atlas de Navarra. Pamplona: Caja de Ahorros de Navarra; 1986, vol. 1, p. 25 y 132; Anaut Bravo, Sagrario. Cambio demográfico y mortalidad en Pamplona (1880-1935). Pamplona: Universidad Pública de Navarra; 1998; Andrés-Gallego, José. Historia contemporánea de Navarra. Pamplona: Ediciones y Libros; 1982, p. 16. 
del siglo XX, especialmente, fue un periodo de dificultades y de penuria por la carestía y escasez de subsistencias ${ }^{16}$.

La Conciliación, como era habitual en estas entidades, hizo constar repetidamente su carácter apolítico, sin embargo, tomó partido en la vida política y social de la ciudad ${ }^{17}$ y contó con el respaldo gubernamental y con ayudas institucionales ${ }^{18}$.

\section{Las fuentes}

Carol Z. Stearns y Peter Stearns han mostrado que es posible examinar la evolución histórica de las normas emocionales de cada sociedad ${ }^{19}$. Aquí nos interesa observar las experiencias emocionales que se suscitan en las fiestas de La Conciliación, para lo cual revisamos cómo quedaron plasmadas en los documentos de su archivo. También contamos con los periódicos de la época, puesto que las actividades de La Conciliación tuvieron un amplio eco en la prensa local. Como hemos mostrado en otro momento, el Diario de Navarra, fundado en 1903, fue órgano de expresión de La Conciliación porque un buen número de socios protectores eran miembros de la junta de accionistas del periódico ${ }^{20}$.

Hasta 1933, La Conciliación estuvo gobernada por una junta mixta. Durante los primeros veinte años, la junta estuvo constituida por dieciocho miembros, seis por cada estamento (obreros, patronos y protectores). A

16. García-Sanz Marcotegui, Angel. El Ayuntamiento de Pamplona ante la «crisis obrera». Gerónimo de Uztariz. 1989; 3: 26-39 (26).

17. León Sanz, Pilar. Networking and interaction between a Mutual Assistance Association and other agencies (Pamplona, 1902-1919). Hygiea internationalis. 2009; 8 (1): 31-50. Característica señalada también por Gabriel, Pere. Sociabilidad obrera y popular y vida política en Cataluña, 1868-1923. Bulletin d'Histoire Contemporaine de l'Espagne. 1993; 17-18: 145-156.

18. León Sanz, Pilar. Private initiatives against social inequalities and health vulnerabilities: the case of La Conciliación (Pamplona, 1902-1920). In: Bourdelais, Patrice; Chircop, John. Vulnerabilities, social inequalities and health. Évora: Ediçoes Colibri; 2010, p. 93-108. Desde 1839 Navarra disponía de una Diputación Foral con competencias en asuntos de bienestar social. El panorama político de Navarra en: Mina Apat, M Cruz. Elecciones y partidos en Navarra (1892-1923). In: García Delgado, José Luis. La España de la Restauración. Política, economía legislación y cultura. Madrid: Siglo Veintiuno; 1985, p. 111-129. Andrés-Gallego, n. 15.

19. Stearns, Peter N.; Stearns, Carol Z. Emotionology. Clarifying the history of emotions and emotional standards. The American Historical Review. 1985; 90 (4): 813-830; Reddy, n. 5, p. 302-304.

20. León-Sanz, n. 17. 
partir de 1922 desaparecieron los patronos ${ }^{21}$. Como en SSM similares, las competencias de la junta mixta eran amplias: resolvían las cuestiones relacionadas con las condiciones y el control de los socorros por enfermedad y la atención médico-farmacéutica; decidían la admisión, expulsión y reingreso de los socios; atendían las reclamaciones y aprobaban las actividades educativas $^{22}$ y recreativas ${ }^{23}$, como los actos del Consultorio para Niños de Pecho y la fiesta anual organizada con motivo de la asamblea general de la Sociedad.

\subsection{Entrega de ropitas a las madres más pobres}

Los médicos de La Conciliación, de forma altruista y con el apoyo de la institución y de las autoridades, pusieron en marcha el Consultorio para Niños de Pecho (1905-1916). Esta iniciativa se enmarca en un movimiento general, en España y en otros países, que trató de disminuir la mortalidad infantil. El consultorio de La Conciliación, además de pesar y medir a los niños, tenía como misión la educación de las madres ${ }^{24}$.

21. León Sanz, n. 10. Andrés-Gallego, n. 9, p. 287.

22. La conexión entre el ámbito de la Historia de la Educación y el de la Historia Social en Guereña, Jean-Louis. La educación popular. In: Guereña, Jean-Louis; Ruiz Berrio, Julio; Tiana Ferrer, Alejandro, eds., Historia de la educación en la España contemporánea: diez años de investigación. Madrid: Centro de Publicaciones del Ministerio de Educación y Ciencia, 1994, p. 141-171. Destaca también esta relación: Solà, Pere. Acerca del modelo asociativo de culturización popular de la restauración. In: Clases populares, cultura, educación. Siglos XIX-XX: coloquio Hispano-Francés. Madrid: Casa de Velázquez; 1990, p. 393-402 (393). Una revisión en: Ruiz Berrio, Julio. La educación social. In: Guereña, Jean-Louis; Ruiz Berrio, Julio; Tiana Ferrer, Alejandro. Nuevas miradas historiográficas sobre la educación en la España de los siglos XIX y XX. Madrid: Ministerio de Educación; 2010, p. 252.

23. Se refieren al tiempo dedicado al ocio y los movimientos obreros: Uría González, Jorge. La cultura popular y la historiografía española contemporánea: breve historia de un desencuentro. In: Ortiz Heras, Manuel; Ruiz González, David; Sánchez Sánchez, Isidro, coords. Movimientos sociales y Estado en la España contemporánea. Cuenca: Universidad de Castilla-La Mancha; 2001, p. 323-377 (359-365); y Navarro, n. 2, p. 474 y 479.

24. León Sanz, n. 17; León Sanz, Pilar. Higienismo y previsión social a través de una Sociedad de Socorros Mutuos: el Consultorio de Niños de Pecho de La Conciliación (1905-1916). In: Castillo, Santiago, Ruzafa, Rafael, coord. La previsión social en la Historia. Vitoria: Instituto de Historia Social Valentín de Foronda; 2008. CD. El Consultorio de Niños de Pecho de La Conciliación corrobora la importancia de las iniciativas filantrópicas privadas señalada en Rodríguez Ocaña, Esteban. Salud pública en España: ciencia, profesión y política, siglos XVIIIXX. Granada: Universidad de Granada; 2005, p. 245. 
Como era habitual en este tipo de establecimientos, a partir de enero de 1906, anualmente, La Conciliación organizó un acto para entregar los donativos a quienes habían demostrado una asistencia más constante y un mayor aprovechamiento de los consejos recibidos en el consultorio ${ }^{25}$. A la celebración asistían los médicos y representantes de la junta mixta, junto con diversas autoridades y la directora, profesoras y alumnas de la Escuela Normal, que colaboraban en la confección de las ropas.

\subsection{La fiesta de La Conciliación}

El día de la Ascensión u otro día festivo del mes de mayo se celebraba «la fiesta de La Conciliación». La junta mixta preparaba con atención el acontecimiento que constaba de «actos religiosos y profanos procurando que éstos últimos tuvieran carácter familiar, culto, educador y benéfico» ${ }^{26}$.

A lo largo de los años, «la fiesta» mantuvo una estructura similar: el día se iniciaba a las siete de la mañana, con la celebración de la santa Misa. A media mañana, en la sede de la sociedad se hacía el reparto de dinero, procedente de donativos a los socios obreros que «pasaban entre los más necesitados» ${ }^{27}$. Normalmente, aquellos que estaban en paro o llevaban más de seis meses de baja por enfermedad, por lo que ya no percibían el subsidio correspondiente.

Posteriormente, tenía lugar el acto central: la junta general de la sociedad con la lectura de la memoria, de las cuentas anuales y la exposición de los proyectos de La Conciliación; seguida de una conferencia de alguna

25. Estas fiestas se generalizaron en la tercera década del siglo XX y se llamaron en algunos lugares, Día del Niño. Rodríguez Ocaña, n. 24, p. 248.

26. Libro de Actas de la Junta Mixta. Archivo de La Conciliación, Universidad de Navarra (en adelante LAC), caja 1; vol. 1; 1903, hojas 61-63. Aunque la historia de la animación sociocultural en España se refiere a un tiempo posterior, señalan las raíces en la educación popular: Úcar Martínez, Xavier, La animación sociocultural. Barcelona: CEAC, 2001; Hernández Díaz, José María. Antecedentes y desarrollo histórico de la animación sociocultural en España. In: Trilla, Jaume, coord. Animación sociocultural: teorías, programas y ámbitos. Barcelona: Ariel; 1997, p. 61-80 (72-73). Son numerosos los estudios sobre la organización de conferencias, veladas teatrales y musicales, por parte de las SSM: Guereña, 2005, n. 7, p. 131-133. Solà, Pere. L'associacionisme obrer a la historia de la societat catalana. L'Avenç. Barcelona. 1993; 171: 28-31; Itineraris per la sociabilitat meridional catalana: Associacionisme i la cultura popular a la demarcació de Tarragona (1868-1964). Tarragona: Diputació de Tarragona; 1998.

27. LAC, caja 1; vol. 1; 1903, hoja 63. 
personalidad. En algunos casos (por ejemplo, en 1927, con motivo de las bodas de plata) se organizaba una comida para todos los socios. Por la tarde, se preparaba una velada que incluía algún concierto o recital musical, escenas de obras de teatro, representadas por socios, etc. La crónica de la fiesta celebrada en 1905 refiere:

«Dio principio la velada con una sinfonía por la orquesta. Los otros números fueron la fábula "La filosofía del vino", representada por varios socios. Intermedio por la orquesta. Y la zarzuela "Crimen misterioso", desempeñada por varios socios; que fue muy bien interpretada por los jóvenes que tomaron parte ${ }^{28}$.

La velada de 1909 se celebró con arreglo al siguiente programa:

«l Sinfonía por la orquesta. $2^{\circ}$ Juguete cómico en un acto titulado "Noche toledana" representado por varios socios. $3^{\circ}$ Aria de barítono, por don José Osés, con acompañamiento de piano. $4^{\circ}$ Intermedio por la orquesta. $5^{\circ}$ Cuento en un acto titulado "Las Olivas", e interpretado por varios socios. Todos los actores estuvieron muy bien: para ellos el señor Osés y el quinteto dirigido por el amigo Aldaba, nuestro sincero aplauso» ${ }^{29}$.

La función de 1916 pasó a llamarse «velada lírico-gráfica» ${ }^{30}$. En 1928, se proyectaron «películas de cine» para los socios y sus familias en el domicilio social de La Conciliación ${ }^{31}$.

\section{Un análisis desde la historia de las emociones}

Las fiestas añaden a las actividades cotidianas un sentido de celebración y de ocio $^{32}$. Como señaló Huizinga, «no es posible la cultura sin una cierta

28. Diario de Navarra. 30 May 1905, p. 1.

29. Diario de Navarra. 11 May 1909, p. 1.

30. Diario de Navarra. 13 May 1916, p. 1.

31. Diario de Navarra. 5 Jun 1928, p. 4.

32. Uría, Jorge. El nacimiento del ocio contemporáneo. Algunas reflexiones sobre el caso español. In: Verdú Maciá, Vicente, et al. Fiesta, juego y ocio en la historia: XIV Jornadas de Estudios Históricos organizadas por el Departamento de Historia Medieval, Moderna y Contemporánea. Salamanca: Ediciones Universidad de Salamanca; 2003, p. 347-382. 
afirmación de la actitud lúdica» ${ }^{33}$. En general, las fiestas conllevan la voluntad de celebrar de quienes participan en ellas ${ }^{34}$.

Por su parte, la historia de las emociones sostiene que las emociones individuales y colectivas se insertan en las estructuras sociales y en las relaciones interpersonales y son parte de un mundo emocional más amplio ${ }^{35}$. Se han acuñado diversos términos para ejemplificar este arraigo de las emociones individuales o colectivas: structures offeelings (Raymond Williams), emotionology (Peter N. Stearns and Carol Z. Stearns), emotional regimes (William M. Reddy) and emotional communities (Barbara H. Rosenwein) por citar los más importantes. William Reddy propone el término emotive para explicar que las expresiones emocionales pueden servir para que una persona opte entre varias reacciones emocionales posibles ${ }^{36}$.

Así se pueden analizar las relaciones entre lo individual y lo colectivo de un modo nuevo; como indica Susan J. Matt, en el ámbito de la historia social:

«[...] los académicos, primero se fijaron en la historia de la clase obrera, luego en las cuestiones de género y raza, y, finalmente, en los intercambios, en las prácticas y en los hábitos de la vida diaria» ${ }^{37}$.

33. Huizinga, Johan. Homo ludens. Madrid: Alianza; 1998, p. 123.

34. Pieper, Josef. Una teoría de la fiesta. Madrid: Rialp; 2006.

35. Hoffman, Martin L. Empathy and prosocial behavior. In: Lewis, Michael; Haviland-Jones, Jeannette M.; Feldman Barrett, Lisa, eds. Handbook of emotions. 3a ed., New York: Guilford Press, 2008, p. 440-455. Comenta las diversas aproximaciones a las emociones en el ámbito social Flam, Helena. The transatlantic element in the sociology of emotions. In: Biess, Frank; Gross, Daniel, eds. Science and emotions after 1945: A transatlantic perspective. Chicago: University of Chicago Press; 2014, p. 318-341.

36. El término Structure of feelings describe los sentimientos como una forma de organización de las experiencias sociales comunes a una generación o grupo social en un momento determinado. Williams, Raymond C. Marxism and Literature. Oxford: Oxford University Press; 1988. La noción de emotionology trata de capturar las expresiones de las emociones y su duración en sociedades guiadas por ciertas normas, Stearns; Stearns, n. 19. Basado en una investigación antropológica y psicológica-cognitiva, William M. Reddy destacó el aspecto performativo de las emociones y describió los «regímenes emocionales». Reddy, William M. Against constructionism. The historical ethnography of emotions. Current Anthropology. 1997; 38 (3): 327-351. Rosenwein ha mostrado que los «grupos sociales» como las familias, los partidos políticos o los gremios crean systems of feeling. Las llamadas emotional communities pueden existir junto con otros grupos sociales o políticos. Rosenwein, Barbara H. Worrying about emotions in history. The American Historical Review. 2002; 107 (3): 821-845.

37. Matt, n. 1, p. 118. 
La perspectiva emocional no necesariamente tiene que ser entendida como una investigación de cuestiones de causalidad o motivación, puesto que las emociones, además, son una parte de las acciones en si mismas, que las acompañan y moldean. Es decir, las emociones no dan tanto una respuesta a la pregunta de por qué se hace algo, sino a la pregunta de cómo se hace algo y lo que significa esa acción. Por eso, aplicamos también el concepto de emotional habitus que incorpora una disposición emocional del que derivan las «prácticas emocionales» definidas por Monique Scheer ${ }^{38}$. Este enfoque tiene en cuenta el concepto de habitus bourdeuliano que plantea que, junto a las normas establecidas o la lógica o el cálculo racionales existen otros principios generadores de la práctica. El habitus sería la capacidad cognitiva, socialmente adquirida, de un sistema o conjunto de disposiciones y que, a su vez, es principio o matriz generador de percepciones, apreciaciones y acciones ${ }^{39}$.

Veremos que el emotional habitus subraya la función de las emociones a través de las prácticas y al hacerlo, tiene en cuenta la dimensión performativa de las emociones en el ámbito social y cultural $^{40}$. Las prácticas de la sociabilidad de La Conciliación fueron cauce y expresión de sentimientos individuales y colectivos. Y siguiendo las categorías expresadas por Monique Scheer en el estudio citado, observaremos que lo emocional «moviliza» a las personas, «escenifica» las actuaciones, sirve como medio de «comunicación» y, ya se ha comentado, contribuye a la «reglamentación ${ }^{41}$.

\subsection{Comunicar y movilizar}

El aspecto «comunicativo» constituye una de las facetas más evidentes de las prácticas emocionales: emociones y sentimientos están involucradas en los procesos de comunicación que, al mismo tiempo, son medio de intercambio emocional $^{42}$.

38. Scheer, Monique. Are emotions a kind of practice (and is that what makes them have a history)? A Bourdieuan approach to defining emotion. History and Theory. 2012; 51: 193-220. Comenta la importancia de las «prácticas emocionales». Pampler, 2014, n. 1, p. 24.

39. Bourdieu, Pierre. Poder, derecho y clases sociales. Bilbao: Desclée de Brouwer; 2000, p. 25, 136.

40. Scheer, n. 38.

41. Scheer, n. 38, p. 193-220.

42. Reddy, n. 5, p. 312. 
En el caso de los actos organizados por el Consultorio de Niños de Pecho interesa resaltar el tono hiperbólico de las expresiones utilizadas en las crónicas: las madres «salían agradecidísimas»; para la sociedad era una «satisfacción» la entrega de las ropitas; los médicos daban «las más expresivas gracias»; y el premio de los donantes era «el agradecimiento de muchas madres de familia». Incluso se habla de «la grata impresión» que el acto causaba en los espectadores ${ }^{43}$.

Las noticias publicadas utilizan un vocabulario que subraya la idea de lo femenino y la función de la mujer en la sociedad de la época. Así, leemos expresiones como esas «pobres» madres, que eran «premiadas»y puestas como modelo por su «celo» en el cumplimiento «solícito» de los deberes como madre. Y también se habla de «los generosos sentimientos femeninos» y de un «corazón maternal» cuando se hace referencia a la confección de ropas por parte de las «señoras y señoritas». Labores que eran realizadas «en sus ratos de ocio».

La crónica del Diario de Navarra sobre el acto celebrado en enero de 1914 puede servir de ejemplo:

«El director del Consultorio don Agustín Lazcano a quien acompañaban los médicos señores Sanz y Lizarraga (don Saturnino) iba leyendo los nombres de los niños y cada madre sacaba una papeleta que tenía el número de uno de los 42 lotes, y don Pedro Uranga [en aquel momento Presidente de La Conciliación] entregaba las ropitas que les habían correspondido. No hay para qué decir que todas las madres salían agradecidísimas» ${ }^{44}$.

Con el tiempo, aparecen en la prensa los nombres de las donantes y las prendas donadas. Sirva de ejemplo el siguiente suelto del 28 de diciembre de 1912:

«Han sido donadas para los niños que acuden al Consultorio de $\mathrm{La}$ Conciliación las siguientes prendas: La Excma. marquesa de Guinior: 12 camisas, 12 pares de mitones, 12 confortantes, 12 gorras, 12 jubones de piqué, 12 jubones de lana, 6 pañales, 6 fajas, 6 pares de medias, 8 mantillas, 4 toquillas y 3 refajos de lino. La señora de Gaztelu: 6 camisas, 6 pañales, 4 mantillas y 6 chaquetas de lino» ${ }^{4}$.

\footnotetext{
43. Diario de Navarra. 10 Sep 1913, p. 2.

44. Diario de Navarra. 8 Ene 1914, p. 1.

45. Diario de Navarra. 28 Dic 1912, p. 3.
} 
También encontramos en las actas y en la prensa el agradecimiento de la sociedad por los donativos monetarios que recibe el Consultorio. En 1906 Agustín Lazcano publicó una carta dirigida al Presidente del festival de la Prensa para dar «las más expresivas gracias por las quinientas pesetas que me ha entregado para el Consultorio de Niños de Pecho que tengo el honor de dirigir» ${ }^{46}$. En otras ocasiones, el donante permanecía en el anonimato:

«El reputado facultativo don Agustín Lazcano, médico de La Conciliación ha recibido de una persona que oculta su nombre, la cantidad de 250 pesetas con destino al Consultorio de niños. A elogios merecidísimos se hace acreedora la persona caritativa que tan espléndidamente socorre a las madres que acuden al Consultorio» ${ }^{47}$.

Estos ejemplos evidencian una emotividad colectiva de satisfacción y gratitud. Además, en las fiestas del Consultorio de Niños de Pecho, lo emocional «movilizaba» a los individuos y a los colectivos: el eco de estos actos en la prensa se prolongaba durante varios días y los médicos aprovechaban para insistir en los consejos puericultores, como la lactancia materna. Las noticias servían, además, para sensibilizar a la sociedad y fomentar los sentimientos de piedad y caridad ante las necesidades de los niños y de las madres pobres. Era un modo de solicitar el apoyo de una población que cada año se volcaba con la entrega de más ropas y donativos:

«Las pobres mujeres que sobre atendérseles gratuitamente con prescripciones para la lactancia de sus hijitos, veían premiados su asiduidad y su celo en el cumplimiento de sus deberes de madre solícita, no encontraban frases de agradecimiento para las señoras y señoritas que habían tenido un recuerdo para los niños pobres. A los generosos sentimientos femeninos acudimos hoy en súplica de que no echen al olvido el Consultorio (...). Su premio será el agradecimiento de muchas madres de familia; agradecimiento de corazón maternal» ${ }^{48}$.

En la actividad del Consultorio de Niños podemos observar la interacción y la cooperación entre La Conciliación y las autoridades, por una parte, y de toda la población de la ciudad, que era convocada para colaborar y participar en las fiestas con fecha y programa organizados.

\footnotetext{
46. Diario de Navarra. 19 Ag 1906, p. 1.

47. Diario de Navarra. 7 Feb 1908, p. 1; 10 Mar 1908, p. 3.

48. Diario de Navarra. 7 Feb 1908, p. 1.
} 


\subsubsection{Las conferencias de las fiestas}

Los conferenciantes invitados eran conocidos políticos y activistas socialcristianos de la España del momento, por lo que las fiestas sirvieron para «movilizar» redes de relaciones interpersonales que tuvieron especial importancia en la marcha de los asuntos de La Conciliación. El prolongado apoyo de estos socios contribuyó a la pervivencia de la institución y a las dinámicas de sociabilidad aquí descritas ${ }^{49}$.

En 1903, para la celebración de la fiesta anual fue invitado Juan Cancio Mena $^{50}$, carlista, casado con Francisca Sarasate, hermana del famoso violinista navarro. Otros invitados fueron el académico de la Real Academia de Ciencias de Madrid, Francisco de Paula Arrillaga ${ }^{51}$; el director del periódico La Gaceta del Norte de Bilbao, José María de Urquijo; el ex-ministro Antonio Goicoechea ${ }^{52}$, etc.

A las fiestas de los años veinte, acudieron, entre otros, Ignacio Navarro, magistrado de Cádiz ${ }^{53}$; Félix Arrarás, magistral de la Catedral de Burgos ${ }^{54}$; José María Gil Robles ${ }^{55}$, entonces profesor de Derecho Político. También aparecieron en la tribuna personalidades pamplonesas como: Joaquín Beunza, abogado pamplonés, concejal y diputado foral de filiación carlista; Daniel Nagore, director del Departamento de Agricultura de la Diputación navarra; Justo Garran y Mosso, abogado, periodista y político, que fue presidente de La Conciliación en $1931^{56}$.

Con frecuencia, las conferencias hacían referencia a aspectos de la teoría del catolicismo social. Así lo refleja el Diario de Navarra con ocasión de la visita de Víctor Pradera:

49. León Sanz, n. 17; Andrés-Gallego, n. 9, p. 12; Andrés-Gallego, José. Navarra, cien años de historia. Pamplona: Diario de Navarra; 2003, p. 49; Goñi Gaztambide, n. 9, p. 191. Comenta el fracaso de una institución similar por la falta de apoyos sociales: Castrillejo Ibáñez, Félix M. El Círculo Católico de Burgos como modelo de sociabilidad confesional (1883-1936). In: Maza Zorrilla, n. 5, p. 237-262 (245).

50. LAC, caja 1; vol. 1; 1903, hojas 61-63; LAC, caja 1, vol. 1; 1903, hojas 316-317.

51. LAC, caja 1, vol. 1; 1903, hojas 304-306.

52. Diario de Navarra. 13 May 1916, p. 1.

53. Diario de Navarra. 6 Jun 1923, p. 6.

54. Diario de Navarra. 26 May 1925, p. 2.

55. Diario de Navarra. 1 Jun 1926, p. 2.

56. Un análisis de la filiación de los conferenciantes: León Sanz, Pilar. The strategies of interrelations between Assistance Associations and other agencies in Pamplona, 1902-1936. In: Health institutions at the origin of the welfare systems in Europe. Pamplona: Eunsa; 2010, p. 167-192. 
«[...] a quién se invitó para que pronunciara en la función de la noche el discurso de apertura de la velada, que como materia más a propósito versa todos los años sobre cuestiones sociales y económicas dentro de las sanas y puras doctrinas de la Iglesia ${ }^{57}$.

Aunque, en esta ocasión, Víctor Pradera trató sobre «La higiene de las grandes ciudades» ${ }^{58}$.

Otras conferencias tuvieron como finalidad promover la puesta en marcha de nuevos beneficios sociales en La Conciliación. Así, se organizaron conferencias sobre las ventajas que suponía la cooperativa de la sociedad ${ }^{59}$ y se encargó a Álvaro López Núñez, secretario del Instituto Nacional de Previsión, que disertara sobre «las pensiones para los obreros en su ancianidad ${ }^{60}$.

\subsection{Escenificar}

Los actos descritos son prácticas imbuidas de temporalidad, por lo que, contestando a uno de los interrogantes de la introducción, podemos afirmar que la historia de las emociones contribuye a la descripción de los estilos emocionales pasados y a la comprensión de su evolución histórica ${ }^{61}$. El paso de las veladas musicales y teatrales a las «lírico-gráficas» y luego a las cinematográficas confirma la evolución de los hábitos sociales respecto a al uso del tiempo libre y la aparición de nuevas prácticas de sociabilidad entre la clase obrera ${ }^{62}$. Corrobora la apreciación de Agulhon acerca de la apropiación por parte de las clases populares de modelos asociativos de clases superiores $^{63}$. Son, al mismo tiempo, un mecanismo de conservación

57. Diario de Navarra. 30 May 1905, p. 1. Mérida-Nicolich, Eloisa. Acción educativa y acción social en España (1889-1912). In: López, Teodoro, ed. Doctrina social de la Iglesia y realidad socioeconómica: en el centenario de la Rerum Novarum. Pamplona: Ediciones Universidad de Navarra; 1991, p. 239-249.

58. Diario de Navarra. 30 May 1905, p. 1.

59. Diario de Navarra. 24 Jul 1917, p. 1.

60. Diario de Navarra. 13 May 1916, p. 1.

61. Reddy, n. 5, p. 312; Scheer, n. 8, p. 201; Lemmings, David; Brooks, Ann, eds. Emotions and social change: Historical and sociological perspectives. New York; 2014, Parte I: «ldentifying emotional communities».

62. Navarro, n. 2, p. 481.

63. Agulhon, Maurice. Classe ouvrière et sociabilité avant 1848. In: Histoire vagabonde. I. Ethnologie et politique dans la France contemporaine. Paris: Éditions Gallimard; 1988, p. 60-97. 
de formas de vida tradicionales, y de acomodación o aprendizaje de nuevas formas de comportamiento ${ }^{64}$.

\subsubsection{Los lugares de celebración}

La historiografía de la sociabilidad ha dado importancia al marco urbano ${ }^{65}$ y también a los lugares concretos donde los socios de las SSM se reunían, ya que, además de servir para resolver cuestiones de tipo laboral y de gestión de las sociedades, eran espacios de «relación y de sociabilidad obrera» que servían para la difusión de principios ideológicos; también se utilizaban para atender las demandas educativas y de ocio de los socios ${ }^{66}$.

Los lugares estuvieron ligados a la escenificación de las fiestas y tuvieron un significativo componente emocional. En el caso de «la fiesta de La Conciliación», las ceremonias litúrgicas tenían lugar en templos señalados de la ciudad: en la capilla de San Fermín, situada en la parroquia de San Lorenzo; o bien en la céntrica parroquia de San Nicolás.

Ese mismo día o la víspera, una comisión recibía a los oradores en el andén de la estación de tren. Así leemos, en 1909, «una comisión de La Conciliación presidida por el presidente de esta Sociedad, D. Rafael Gaztelu [recibió] al señor Posse, redactor jefe de La Gaceta del Norte» ${ }^{67}$.

Los actos tenían lugar en locales emblemáticos: el Teatro Gayarre (antiguo Teatro Principal) o las Escuelas de San Francisco de la capital navarra, donde la reunión se celebraba «con gran bombo y solemnidad». Las crónicas cuentan que «el interior del teatro ofrecía un aspecto deslumbrador; todas las localidades estaban ocupadas, y la iluminación era profusa» 68 .

El desarrollo de los actos incluía una verdadera puesta en escena: en el teatro se levantaba el telón que mostraba el escenario con la mesa constituida por el presidente y otros representantes de la junta mixta y el orador invitado, a ellos se unió en ocasiones el obispo (figura 1). Se

\footnotetext{
64. Escalera Reyes, Javier. Asociacionismo y Antropología. In: Maza Zorrilla, n. 5, p. 9-20 (11).

65. Erice Sebares, Francisco. La clase obrera española en el siglo XIX: balance y perspectivas historiográficas. In: Ortiz; Ruiz; Sánchez, n. 23, p. 45-79. Destaca la consolidación de una cultura urbana en este período: Navarro, n. 2, p. 482.

66. Navarro, n. 2, p. 478; Gabriel, n. 17, p. 154. Guereña; Tiana, n. 22 1994, p. 158. Como señala Guereña 2005, n. 7, p. 88, no se trataba de espacios neutros vacíos de contenido ideológico.

67. Diario de Navarra. 11 May 1909, p. 1. Se trata de José de Posse y Villelga.

68. Diario de Navarra. 30 May 1905, p. 1.
} 
cuidaba con esmero el protocolo y el orden en el que aparecían ocupados los asientos y «destacándose al fondo del palco escénico la hermosísima bandera de la Sociedad ${ }^{69}$. Este modo de proceder favorecía la formalidad y la expectación. El inicio del acto era recibido con una «nutrida y prolongada salva de aplausos» por parte de los asistentes ${ }^{70}$.



Figura 1. Fiesta de La Conciliación de 1926 celebrada en el Teatro Gayarre (Foto Galle, Archivo Municipal de Pamplona).

A las fiestas debían acudir todos los socios de La Conciliación, y en efecto, la asistencia, también al acto religioso, parece que era elevada. Así, la crónica de 1909 refiere que «la espaciosa capilla contenía una multitud incalculable de fieles que de manos del digno capellán de la Sociedad recibieron el Pan de los Ángeles, con gran fervor religioso» ${ }^{71}$. También parece que estaba completo el aforo del teatro:

69. Diario de Navarra. 1 Jun 1926, p. 2.

70. Diario de Navarra. 30 May 1905, p. 1; 11 May 1909, p. 1.

71. Diario de Navarra. 11 May 1909, p. 1. 
«A los breves instantes de haber sido abiertas las puertas del Teatro Gayarre, se hallaba éste de bote en bote: todas las localidades fueron ocupadas y no pocos socios hubieron de presenciar la velada de pie en palcos, anfiteatro y hasta intercalados en el patio de butacas» ${ }^{72}$.

Se ha descrito el carácter interclasista e integrador de estas iniciativas ${ }^{73}$, y en el caso de una sociedad mixta, como La Conciliación, este aspecto estaba cargado de significado y fue subrayado en la prensa:

«Al lado de un capitalista se veía un bracero: junto a una levita, una blusa: una boina al lado de un sombrero. Allí estaban hermanados el trabajo y el capital, el rico y el pobre, el ignorante y el sabio (...) allí no habla diferencia de clases ni distinción de condiciones» ${ }^{74}$.

Cada año, la prensa se hacía eco de lo «brillantísimas y solemnes y animadas» que habían resultado las celebraciones. En 1909 aseguraban que «no había decaído nada del esplendor que revestía en años anteriores» ${ }^{75}$. También reiteran que los oradores eran de gran «elocuencia», «sencillez» y «elegancia». Por lo que no es de extrañar que las disertaciones fueran precedidas y seguidas de gran entusiasmo:

«La presencia del señor Posse en el palco escénico fue acogida con una nutridísima y prolongadísima salva de aplausos: fue una espontánea manifestación de cariño y simpatía que el obrero católico de Pamplona tributaba a su nuevo maestro» ${ }^{76}$.

La conferencia de Víctor Pradera fue seguida de «espontáneas y estruendosas salvas de aplausos que por sí solas bastan para demostrar la simpatía que su juventud y ciencia inspiraron al público» ${ }^{77}$. En la crónica

72. Diario de Navarra. 11 May 1909, p. 1.

73. Uría, Jorge. La cultura popular en la Restauración. El declive de un mundo tradicional y el desarrollo de una sociedad de masas. In: Suárez Cortina, Manuel, ed. La cultura española en la Restauración. Santander: Sociedad Menéndez Pelayo; 1999, p. 125; Uría, Jorge. Una historia social del ocio. Asturias, 1898-1914. Madrid: Publicaciones Unión-Centro de Estudios Históricos; 1996, pp. 254-255.

74. Diario de Navarra. 18 May 1917, p. 2.

75. Diario de Navarra. 11 May 1909, p. 1.

76. Diario de Navarra. 11 May 1909, p. 1.

77. Diario de Navarra. 30 May 1905, p. 1. 
del 1 de junio de 1926 leemos: «Al terminar su discurso, bello en la forma y hermoso en el fondo, el señor Gil Robles oyó una ovación delirante y muy prolongada». Los discursos de los miembros de la junta mixta y del señor obispo también eran «aplaudidísimos» ${ }^{78}$.

Con el tiempo es posible que disminuyera la afluencia porque, en 1928, se propuso agregar al programa una rifa de premios (una gratificación de doce pesetas) entre los socios presentes en el acto. El número de premios fue variando con los años (en 1928, se repartieron 20 premios, en 1929, 40 y en 1930 fueron 50 $)^{79}$.

En los apuntes recogidos en las actas y en las noticias publicadas en la prensa observamos que estos actos constituyeron también acontecimientos para la ciudad. Así en una crónica del Diario de Navarra se recoge que «A las siete de la tarde los alrededores del Teatro Gayarre estaban repletos de gente que presenciaba la gran afluencia de socios de La Conciliación a nuestro pequeño coliseo ${ }^{80}$.

\subsection{Reglamentar}

Por último, uno de los campos clásicos de la investigación de la emoción, es el análisis de las emociones como instrumento de regulación. Se habla de «reglas o normas emocionales» o emotionology, según el término acuñado por Peter N. Stearns para referirse a las demandas emocionales de los lugares y a las etiquetas sociales relacionadas con la gestión emocional en una sociedad concreta ${ }^{81}$. Por su parte, Ute Frevert refiere que las instituciones - como La Conciliación - juegan un papel esencial en la difusión de las normas y reglas emocionales ${ }^{82}$. En las fiestas de La Conciliación hemos reconocido guías, estándares emocionales. Los actos fueron un

\footnotetext{
78. Diario de Navarra. 1 Jun 1926, p. 2.

79. Orantos Míguez, M. Dolores. La Conciliación (1923-1931). Pamplona: Facultad de Filosofía y Letras, Universidad de Navarra; 1988.

80. Diario de Navarra. 30 May 1905, p. 1.

81. Stearns; Stearns, n. 19, p. 813-836.

82. «Institutions also played a part as essential amplifiers that helped to disseminate emotional norms and rules... Institutions like family, church, school, the military, the workplace and politics served as "schools of emotions" that shaped the self-perception and outlook of generations of respective students». Frevert, Ute. Emotions in history: lost and found. New York: Central European University Press; 2011, p. 210.
} 
ámbito de expresión de sociabilidad y un referente de identificación que integró a los socios obreros, a los socios patronos y a los socios protectores como actores y como población diana ${ }^{83}$ creando así un grupo o comunidad emocionales integrada por quienes componían La Conciliación ${ }^{84}$. En efecto, observamos una importante conciencia de grupo alrededor de las actividades de la Sociedad.

El estudio pone de manifiesto que las personas viven y se mueven en espacios que generan comunidades que comparten estilos y conductas emocionales en los que se comunican y pueden entenderse. A través de los actos descritos se buscaba un tipo de compañerismo, se participaba en concepciones ideológicas, religiosas o políticas. Por ejemplo, aunque desde finales del siglo XIX está descrito en España un proceso de «laicización» entre los obreros que se manifestó en una menor frecuentación de los lugares de práctica religiosa ${ }^{85}$, el caso de La Conciliación muestra que, al menos en Pamplona, lo católico siguió ocupando espacios de sociabilidad en el ámbito obrero durante el primer tercio del siglo XX.

\section{El carácter transformador de las emociones}

Podemos concluir señalando la dificultad que entraña el estudio de las emociones en la historia social. Por una parte, condiciona la comprensión de la sociabilidad y del fenómeno asociativo en sí mismo. Por otra, no resulta fácil definir las reacciones emocionales individuales de los socios puesto que los sentimientos son expresados a través de relatos de terceras personas y de las crónicas de las fiestas. Al mismo tiempo, la investigación confirma el interés de analizar situaciones concretas: una ciudad, una institución, unos socios, unos obreros, una ideología, un momento, unas actividades, ya que todo ello interviene en la identidad del grupo. Como señala M. Ralle, las investigaciones sobre sociabilidad han de acercarse a la realidad, para que no se queden en aproximaciones abstractas o teóricas ${ }^{86}$.

\footnotetext{
83. Ralle, n. 7, p. 162 y 197; Escalera Reyes, n. 64, p. 11.

84. Rosenwein, n. 36, p. 842.

85. Ralle, n. 7, p. 196.

86. Ralle, n. 7, p. 162.
} 
Benno Gammerl ha señalado que los estilos emocionales interactúan en los espacios, interpretando lo público o lo privado ${ }^{87}$. Las fiestas de La Conciliación fueron actos formales, en los que, al mismo tiempo, se querían inculcar «sentimientos familiares». Si, como hemos visto, el habitus de Bordieu se compone de «esquemas de percepción, pensamiento y acción» que producen los actos ${ }^{88}$, aquí observamos que los gestos de los participantes en las fiestas expresan a los individuos, aunque estén socialmente condicionados. A menudo la reacción personal se guía por recuerdos de situaciones o estrategias pasadas, hábitos que siguen su propia lógica en las prácticas cotidianas o periódicas ${ }^{89}$. De ahí el interés de la dimensión «performativa» o transformadora de las emociones, propuesta por William M. Reddy ${ }^{90}$. Como expresaba más recientemente Jeffrey S. Juris, al estudiar las protestas y manifestaciones sociales y laborales, cualquier actividad en el ámbito de los movimientos sociales es performativa ${ }^{91}$. Lo que proporciona una dimensión dinámica al componente emocional en la sociedad.

En nuestro caso, la expectación expresada en la recepción de los conferenciantes en la estación de ferrocarril, la disposición del escenario o la subida del telón en el teatro Gayarre, la acogida de los discursos,... provocaron emociones que contribuyeron a «re-configurar» la identidad de los asistentes. Las actividades de las fiestas proponían al socio obrero y a sus familias un modelo de conducta. Además, las actuaciones de los socios, protectores u obreros, en las fiestas sirvieron para hacer y formar un grupo. Se trata de una sociabilidad al servicio del proyecto de la Sociedad que dio origen a un fuerte sentido identitario que se prolongó a lo largo de los años.

A lo emocional se une lo racional, lo cual permite la adhesión a las estrategias, o a la lógica intencional, orientada a objetivos. Las fuentes evidencian los sentimientos y estilos de vida de los socios obreros, pero también la intencionalidad de las acciones emprendidas por la mutualidad. Las actividades educativas o lúdicas que organizó La Conciliación estaban

87. Gammerl, Benno. Emotional styles-concepts and challenges. Rethinking History. 2012; 16 (2): 161-175.

88. Scheer, n. 38, p. 201

89. Scheer, n. 38, p. 203.

90. Reddy, n. 35.

91. Juris, Jeffrey S. Embodying protest. In: Britta Baumgarten; Priska Daphi, Peter Ullrich, eds. Conceptualizing culture in social movement research. Basingstoke: Palgrave Macmillan; 2014, p. 227-228. Además en ese mismo volumen: Doerr, Nicole. Memory and culture in social movements, p. 206-226. 
directamente relacionadas con la finalidad de la Sociedad y también supusieron un mayor apoyo social e institucional a la entidad.

Como señala M. Scheer, concebir las emociones como «respuestas orientadas a objetivos», ha de evitar pensar en lo «estratégico» como algo superficial ${ }^{92}$. Si bien es cierto que los recursos dedicados al socorro por enfermedad o a la asistencia médico-farmacéutica fueron mucho mayores, no obstante, estos actos no eran una cuestión marginal: respondían al carácter mixto y católico que mantuvo La Conciliación hasta 1933. Lo cual corrobora las aportaciones de otros estudios sobre sociabilidad ${ }^{93}$.

El análisis de la sociabilidad ha permitido también aproximarnos al concepto de «obrero» en una época en la que no había una noción uniforme o unívoca ${ }^{94}$. En el caso de La Conciliación, los socios obreros pasaron a la categoría de patronos y viceversa; algunos obreros eran pobres y otros no. Había obreros especialistas y otros sin formación. Los socios obreros tenían en común el hecho de vivir en la ciudad y la voluntad de asociarse a una organización de esas características, puesto que existían otras, de diferente ideología, que facilitaban prestaciones semejantes.

El modelo de obrero y de institución, objeto de este estudio, se suma a otros muy diferentes, de filiación republicana y socialista, que se desarrollaron en la época. Las emociones descritas en las fiestas de La Conciliación, con los años, formaron «hábitos», rituales y motivo de ocio y pasatiempo. A través de estos actos, se ha dicho, se reproduce la llamada «sociabilidad popular de imitación» ${ }^{95}$.

92. Scheer, n. 38, p. 207

93. González Gómez, Santiago. El asociacionismo obrero en Madrid a principios del siglo XX. Salamanca: Universidad; 1982, p. 9; Solà, Pere. Poderes y asociacionismo en la España contemporánea (siglos XIX y XX). Algunas consideraciones. In: Castillo, Santiago; Ortiz de Orruño, José M, coords. Estado, protesta y movimientos sociales. Bilbao: Universidad del País Vasco; 1998, p. 359-370. Castillo, Santiago, ed. Solidaridad desde abajo: trabajadores y socorros mutuos en la España Contemporánea. Madrid: UGT Centro de Estudios Históricos; 1994; Montero García, Feliciano; Esteban de Vega, Mariano. Aproximación tipológica al mutualismo popular y obrero en España: el mutualismo asistencial. In: Castillo, Santiago, coord. La historia social en España: actualidad y perspectivas. Madrid: Siglo Veintiuno de España; 1991, p. 462; AndrésGallego, n. 9; Montero, Feliciano. Propaganda católica y educación popular en la España de la Restauración. In: Fell, Eve-Marie; Guereña, Jean-Louis. Ecole et société en Espagne et en Amerique Latine (XVIIle-XXe siecles). Tours: Université; 1985, p. 265-279.

94. León Sanz, n. 17; Ralle, n. 7, p. 161.

95. Agulhon, Maurice. La République au village: les populations du Var, de la Révolution à la II République. Paris: Seuil; 1979, p. 207-214; Ralle, n. 7, p. 169. Navarro, n. 2, p. 481 comenta la consolidación de una identidad socialista mediante prácticas de afirmación colectiva. 
Olivier Faure, al tratar del desarrollo de las mutuas francesas a finales del siglo XIX comenta: «La mutualidad era un sueño social y una realidad médica» ${ }^{96}$. Aquí nos hemos referido al primer aspecto y hemos comprobado que, como señala M. Ralle, la construcción de una identidad obrera no es el resultado de un caminar rectilíneo ${ }^{97}$. En el devenir de las ciudades emergieron no una sino diversas culturas obreras ${ }^{98}$, y sus identidades se expresaron en los espacios de la sociabilidad.

96. Faure, Olivier. Les Français et leur médecine au XIXe siècle. Paris: Belin; 1993, p. 121-142.

97. Ralle, n. 7, p. 199.

98. Olabarri Gortazar, Ignacio. ¿Lucha de clases o conflictos de intereses?: ensayos de historia de las relaciones laborales en la edad contemporánea. Pamplona: Ediciones Universidad de Navarra; 1991, p. 161. 